\title{
MABC: Power-Based Location Planning with a Modified ABC Algorithm for 5G Networks
}

\author{
Ruchi Sachan, ${ }^{1}$ Zahid Muhammad, ${ }^{1}$ Jaehoon (Paul) Jeong, ${ }^{2}$ \\ Chang Wook Ahn, ${ }^{3}$ and Hee Yong Youn ${ }^{4}$ \\ ${ }^{1}$ Department of Computer Engineering, Sungkyunkwan University, 2066 Seobu-ro, Jangan-gu, \\ Suwon, Gyeonggi-do 16419, Republic of Korea \\ ${ }^{2}$ Department of Interaction Science, Sungkyunkwan University, 2066 Seobu-ro, Jangan-gu, Suwon, \\ Gyeonggi-do 16419, Republic of Korea \\ ${ }^{3}$ School of Electrical Engineering and Computer Science, Gwangju Institute of Science and Technology (GIST), \\ 123 Cheomdangwagi-ro, Buk-gu, Gwangju 61005, Republic of Korea \\ ${ }^{4}$ Department of Computer Engineering, Sungkyunkwan University, Suwon, Republic of Korea
}

Correspondence should be addressed to Jaehoon (Paul) Jeong; pauljeong@skku.edu

Received 9 February 2017; Revised 14 April 2017; Accepted 4 June 2017; Published 6 July 2017

Academic Editor: Kamel Barkaoui

Copyright (C) 2017 Ruchi Sachan et al. This is an open access article distributed under the Creative Commons Attribution License, which permits unrestricted use, distribution, and reproduction in any medium, provided the original work is properly cited.

\begin{abstract}
The modernization of smart devices has emerged in exponential growth in data traffic for a high-capacity wireless network. 5G networks must be capable of handling the excessive stress associated with resource allocation methods for its successful deployment. We also need to take care of the problem of causing energy consumption during the dense deployment process. The dense deployment results in severe power consumption because of fulfilling the demands of the increasing traffic load accommodated by base stations. This paper proposes an improved Artificial Bee Colony (ABC) algorithm which uses the set of variables such as the transmission power and location of each base station (BS) to improve the accuracy of localization of a user equipment (UE) for the efficient energy consumption at BSes. To estimate the optimal configuration of BSes and reduce the power requirement of connected UEs, we enhanced the $\mathrm{ABC}$ algorithm, which is named a Modified $\mathrm{ABC}$ (MABC) algorithm, and compared it with the latest work on Real-Coded Genetic Algorithm (RCGA) and Differential Evolution (DE) algorithm. The proposed algorithm not only determines the optimal coverage of underutilized BSes but also optimizes the power utilization considering the green networks. The performance comparisons of the modified algorithms were conducted to show that the proposed approach has better effectiveness than the legacy algorithms, ABC, RCGA, and DE.
\end{abstract}

\section{Introduction}

An increasing number of mobile devices with data intensive applications are generating an enormous amount of data. Considering the growth of mobile devices in day-to-day life, the future networks must be capable of dealing with the ever-increasing mobile data traffic. Nowadays devices are ubiquitous with an expected cellular subscription of over 4.55 billion worldwide [1]. Most of the devices today support the majority of services like $3 \mathrm{G}$ and $4 \mathrm{G}$-LTE recently, and next, for the future, it must be capable of handling the rise of the critical factors such as excessive data traffic stress along with $5 \mathrm{G}$ networks. Radio access networks almost consume $80 \%$ of the power in cellular networks in recent communication technology due to irregular planning [2]. Moreover, it is noted that base stations (BSes) consume a significant amount of the energy (above 50\%) in cellular networks [3,4]. To estimate the locations of BSes to optimize the transmission power concerning the green aspects is required.

The vision of $5 \mathrm{G}$ wireless communications extends to offer very high data rates, notably low latency, enhanced base station capacity, and significant improvement in users' perceived good quality of service (QoS) compared to current 4G-LTE networks. A quick look into recent wireless network statistics reports that the global mobile traffic experienced around $70 \%$ growth [5] in 2014. Only 26\% smartphones 
(of the total global mobile devices) are responsible for $88 \%$ of total mobile data traffic [5] and more than 50\% energy consumption spent by the BSes [2, 3].

The following information is usually needed to address the network planning problem for systems with $5 \mathrm{G}$ air interface: (1) a set of candidate sites that is required where BSes can be installed, (2) a set of possible configuration setting that is needed for each BS's orientation, height, and maximum power to allocate the location of BSes, (3) traffic distribution parameter which represents the connected users moving around the cell, and (4) propagation radio channel models with allocated frequency which can support the upcoming $5 \mathrm{G}$ wireless networks. The mentioned points above can be used to estimate the accurate information of future users in $5 \mathrm{G}$ wireless network. By installing the sufficient BSes in a possible position based on the user's behavior, we can enhance the power efficiency of the network [6]. Location information was also available in previous generations of cellular networks regarding different perspectives in $2 \mathrm{G}, 3 \mathrm{G}$, and $4 \mathrm{G}$. For instance, cell-ID positioning is used in $2 \mathrm{G}$, timing-based positioning is used in $3 \mathrm{G}$, and dedicated positioning is used in $4 \mathrm{G}$. Even with the above location information, the researchers of $5 \mathrm{G}$ [7] have found that the range from hundreds to tens of meters is insufficient for some communication operations. So for the first time majority of user equipments (UEs) can benefit from the positioning technologies which can achieve location accuracy in the order of one meter. $5 \mathrm{G}$ should be the first generation to get a benefit from position information for wireless network design and optimization.

In this paper, we have modified the Artificial Bee Colony (ABC) algorithm [8] to optimize the location estimation with the minimum power required for the UEs with less activated BSes. Through the comparison of Modified ABC (MABC) with the other mentioned algorithms such as Real-Coded Genetic Algorithm (RCGA) and Differential Evolution (DE) algorithm, we found that even the modified RCGA (MRGA) with the shuffling of their chromosome could not precisely find an optimum solution [4]. However, the standard ABC has performed well, compared to the other implemented techniques, such as RCGA, MRGA, and DE, because of its different methods of playing employed bees and scout bees to optimize the solution efficiently for our targeted problem.

The paper is structured as follows: Section 2 introduces a literature survey which compares our work with other tasks of optimizing the energy consumption in wireless cellular networks. In Section 3, system model presents a description of the general framework for $5 \mathrm{G}$ network planning. Section 4 compares the implementation of the Modified ABC algorithm to the targeted problem with its applications. Section 5 shows the experimental results obtained from our proposed methods with the perspective of $5 \mathrm{G}$ networks aspects. Finally, Section 6 concludes our work along with future work.

\section{Related Work}

Till now, many researchers have studied the network design problems related to planning and performance optimization in cellular networks considering the latest advancements in the technologies. There are many possible ways to find a precise location information in wireless network system along with parameters such as distances, velocities, angles, delays, and predictable user behaviors [7]. In 5G networks, a location awareness system can be engaged in a wide range of ways to address several keys challenges. Due to the ability of network planning to perform the resource allocation by expecting the channel quality apart from the traditional time scale mentioned in the CSI- (channel state information-) based solution, it can reduce the overhead and delay of the location-aware resource allocation techniques.

In [9], Berrocal-Plaza et al. discussed the optimal location-aware configuration issue by using the Evolutionary Algorithms (EAs), such as Genetic Algorithm (GA) and ABC, to efficiently meet the coverage and traffic requirements for the targeted BSes. They aimed to make a GA in [9] into two versions, named FPS-GA (Genetic Algorithm with Fixed Population Size) and APS-GA (Genetic Algorithm with Adaptive Population Size), to minimize the interference among cells and reduce the energy consumption. It is noted that the balanced load could not completely satisfy the UEs by using the FPS-GA and APS-GA. However, the ABC algorithm required less computational efforts than both FPS-GA and APS-GA.

The location area schemes in [10-12] explain the recent developments in the cellular technologies. They partition a network into the multiple regions or location areas, consisting of one or more cells for each region, by updating the performance of the UEs according to the exact optimal locations of the BSes. Another location management scheme is suggested in [13] where a subset of cells in the network are designated as the reporting cells, and each UE performs its location update only when it enters one of the targeted reporting cells. The objective for using the reporting cell is when a call arrives, the search will define the task of the reporting cell which the user has last reported and the neighboring bounded by the nonreporting cells. Taking advantage of targeted reporting cells, the authors have generated the optimized results by using GA, Tabu Search (TS), and Ant Colony Algorithm (ACA) for the location management.

In [4], the authors modified the traditional RGA to make novel GAs for the future generation of cellular networks. As they mentioned that because of shuffling all the chromosome in crossover operation, the performance of standard RGA makes worst solution over the generation. So for solving the network planning problem by making MRGA perform better than RGA, they introduced Box Crossover Rate (BCR) with less shuffling in crossover operation and small standard deviation values and also compared the results with $\mathrm{DE}$ where they use Scaling Factor 0.5 and Crossover Rate 0.9 for DE over the 50 independent runs.

In [14], Ali et al. considered the simultaneous planning of BSes and Relay Stations (RSes) with the link capacity by using EAs. They aimed at finding an optimized set of BSes and RSes that can fulfill the demand of UEs at the lowest cost. Yu et al. in [15] considered a large coverage area that requires high computation time intractable to the network planning problem.

For tackling the issue of sustainable energy consumption, some researchers in $[16,17]$ also examined the methods of 
achieving optimal energy saving by turning off traffic under loaded BSes. In [16], disabling the unwanted cells with low traffic conserves a significant amount of energy, similar to most of the studies for the power saving progress, where most of the researchers tackle the sleeping mode at UEs [17].

\section{System Model}

In this section, we considered a network planning to design the system model which satisfies the area of $[W \times H] \mathrm{km}^{2}$ for both LTE and $5 \mathrm{G}$ networks. In our system model, BSes can be installed at a set of candidate sites $H=\left\{h_{1}, h_{2}, \ldots, h_{M}\right\}$ in the given targeted area. In order to place the BSes, the installation cost is associated with each of the candidate sites such that $C=\left\{c_{1}, c_{2}, \ldots, c_{M}\right\}$. In our experiment, $K$ denotes the number of BSes; the set of BSes is represented as $S=\left\{s_{1}, s_{2}, \ldots, s_{K}\right\}$.

Our aim is to design a network planning process to minimize the power consumption. The transmission power in a range of 0.1 to 10 watts has been considered as a transmitter attribute for our optimization algorithm. However, the value of antenna gain depends on the manufacture, but we have considered the antenna gain as $18 \mathrm{dBi}$ and frequency as $1800 \mathrm{MHz}[18,19]$. We employ the radio propagation model also known as Cost-231 HATA urban propagation model which enlarges the urban HATA model to cover a more expanded range of frequencies $[8,20]$. In (1), the Signalto-Interference-plus-Noise Ratio (SINR) value is calculated, where the coverage probability in the given area around the location $h_{i}$ with a threshold is less than the SINR value. $M_{g}$ represents the Mast Head Amplifier (MHA) gain, $P_{t}$ represents the transmission power, and $I$ and $N$ account for the interference and noise, sequentially:

$$
\operatorname{SINR}=\frac{M_{g} \times P_{t}}{I+N} .
$$

After getting the value of SINR, the path loss (PL) is determined by

$$
\mathrm{PL}[\mathrm{dB}]=P_{t}+G_{t}-L_{b}-\operatorname{SINR}
$$

where $G_{t}$ represents the antenna gain of the transmitter and $L_{b}$ represents the body loss in $\mathrm{dB}$. Furthermore, the coverage area of a BS is expressed by

$$
\mathrm{CA}_{b_{i}}=\sqrt[3]{3}\left(\frac{R^{2}}{2}\right)
$$

where $R$ represents the cell radius. The coverage probability in the given area around the location $h_{i}$ having threshold $T$ is defined in

$$
P_{c}\left(h_{i}\right)=P\left(\operatorname{SINR}\left(h_{i}\right)>T\right) .
$$

\section{The Proposed Algorithm}

The application of EAs, namely, GA, DE, RCGA, and ABC, is a stochastic exploration search method to resolve both constrained and unconstrained optimization problems, which originates from the natural selection. In this terminology, an individual is referred to as a candidate solution to the targeted optimization problem. These algorithms deal with a set of individuals during their process called the population. ABC is one of the efficient applications of EAs which we have applied and we extended the traditional $\mathrm{ABC}$ to a Modified Artificial Bee Colony (MABC) and compared with our previous work of modified RCGA called MRGA. RCGA contains a continuous decision variable where the GA contains a binary coded variable that is a primary difference between GA and RCGA. The application of EAs gives satisfactory solutions to NP-hard problems. Additionally, EAs are also used to solve many practical problems, such as the finding of an optimal position for a BS in an explicitly particular area of interest $[21,22]$.

4.1. Encoding. The fundamental design of chromosomes is a primary phase of any application of EAs. A chromosome is a set of parameters which specify a proposed solution to the targeted problem that the algorithm is trying to resolve. Usually, a set of chromosomes is a possible settlement for gaining a better representation of an optimal solution of any NP-hard problem.

This paper defines the available transmit power $\left(Q_{i}\right)$ and location of a $\mathrm{BS}\left(X_{i}, Y_{i}\right)$ as a decision variable to the target problem. Here we have used only a constant value for representing a chromosome. The solution of the targeted problem uses these decision variables for chromosomes as described in the following list:

\section{Decision Variables}

$Q_{i}$ : available transmit power of a base station $s_{i}[0.1$ to 10.0] Watt

$X_{i}$ : location of a base station $s_{i}$ in $x$-axis

$Y_{i}$ : location of a base station $s_{i}$ in $y$-axis.

The structure of chromosome is presented in Figure 1 where a set of the populations represents a generation. The population consists of a set of $P$ individuals. In general, a set of individuals is called a population in EAs. Each individual is composed of $K$ BSes where one BS has three decision variables such as its power $\left(Q_{i}\right)$, location- $X\left(X_{i}\right)$, and location- $Y\left(Y_{i}\right)$.

4.2. Artificial Bee Colony. A new and recent application of EAs defines $\mathrm{ABC}$ as a swarm intelligence algorithm which is driven by the behavior of honey bees. ABC simulates the intelligent foraging behavior of real honey bees on finding food positions for their nectar source. ABC algorithm contains three groups of bees: employed bees, onlooker bees, and scout bees. The employed bees have always got a chance to start searching for food around the given food source in their memory, and then they share the information about these food sources with the onlooker bees. The onlooker bees get the chance to select good food sources during sharing information by employed bees. The higher quality of the food source has a significant chance to be chosen by the onlooker bees. The scout bees convert from a few employed bees that abandon their food sources in the process and search for new ones [23]. 


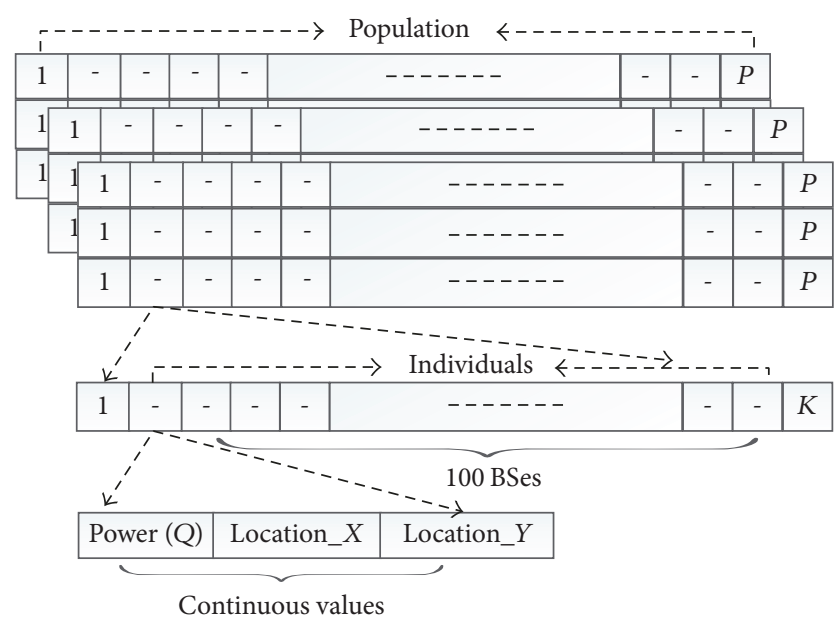

Figure 1: The structure of chromosomes.

In our algorithm, a food source position signifies a possible solution to the optimization problem as an individual in the population, and the nectar quality resembles their fitness function value. The general procedure of $\mathrm{ABC}$ is described in the following points.

4.2.1. Initialization of the Population. The initial phase of $\mathrm{ABC}$ algorithm first generates an initial population randomly according to a uniform distribution within a possible space. The BS axes $(X, Y)$ are represented as a configuration with having the ranges of power $Q=\left[Q_{\min }, Q_{\max }\right]$. Here $Q_{\min }$ and $Q_{\max }$ are the minimum and maximum power required to each BS in the unit of watts. Consider, for $\mathrm{ABC}, X=$ $\{1,2, \ldots, D\}$ and $Y=\{1,2, \ldots, D\}$, such as two series of length vector $D$ with required power range $\left[Q_{\min }, Q_{\max }\right]$. Forming the two-time series pairwise results in a set, $S=$ $\left\{s_{1}, s_{2}, \ldots, s_{D}, Q\right\}$, where $S_{i}=\left(X_{i}, Y_{i}\right)$. Rearrange BSes according to the values of $X_{i}$ and $Y_{i}$. It gets an order set like $S_{i}=$ $\left\{\left(X_{1}, Y_{1}\right),\left(X_{2}, Y_{2}\right), \ldots,\left(X_{D}, Y_{D}\right), Q_{D}\right\}$. All these values are bounded within the range of a lower bound to an upper bound with the random number between $\operatorname{rand}(0,1)$ during the initialization of the population.

An $A B C$ procedure produces a uniformly distributed population of solutions of $P$ number where each solution refers to the solution of each decision variable taken in our simulation. The solution in our simulation represents BSes as $S_{i}=\{i=1,2, \ldots, P\}$ and users as $U_{i}=\{i=1,2, \ldots, P\}$ with a given $D$-dimensional vector. Here $D$ is the number of variables to be optimized as $j \in\{1,2, \ldots, D\}$, and $S_{i}$ and $U_{i}$ represent the $i$ th food source in the population. The BS $S_{i}$ and the user $U_{i}$ are generated as follows in (5) and (6):

$$
S_{i}^{j}=S_{\mathrm{LB}}^{j}+\operatorname{rand}(0,1)\left(S_{\mathrm{UB}}^{j}-S_{\mathrm{LB}}^{j}\right),
$$

where $S_{\mathrm{LB}}^{j}$ and $S_{\mathrm{UB}}^{j}$ are the lower bounds and upper bounds of BSes $S_{i}$ in the $j$ th directions.

$$
U_{i}^{j}=U_{\mathrm{LB}}^{j}+\operatorname{rand}(0,1)\left(U_{\mathrm{UB}}^{j}-U_{\mathrm{LB}}^{j}\right),
$$

where $U_{\mathrm{LB}}^{j}$ and $U_{\mathrm{UB}}^{j}$ are the lower bound and upper bound of given the user $U_{i}$ in the $j$ th directions.
4.2.2. Employed Bees Phase. In the employed bee phase, employed bees modify the current solution obtained from the neighborhood of the current food source based on the information of individual experiences, and their fitness values mean nectar amount of the new solution. The bee updates their position $A_{i}$ by replacing the old one solution of $S_{i}$ and $U_{i}$. If the fitness value of the new food source is higher than that of the old food source, the updated solution of an $i$ th candidate in this phase is shown in as follows:

$$
A_{i}^{j}=S_{i}^{j}+\phi_{i}^{j}\left(S_{i}^{j}-S_{k}^{j}\right)
$$

where $A_{i}^{j}$ is a new solution of the $S_{i}$ and $k$ indicates the $k$ th candidate solution index randomly selected from a candidate solution which must be different from the $i$ th candidate solution. $k \in\{1,2, \ldots, P\}, i \in\{1,2, \ldots, P\}$, and $j \in$ $\{1,2, \ldots, D\}$ are three randomly chosen indices. $\phi_{i j}$ generates a random number within $[-1,1]$ with a uniform distribution.

An example of the base station's position update process in the employed bee phase is described in Figure 2. Firstly, the current state of bee is represented as $S_{i}$ and the highlighted box represents the randomly picked direction $j . S_{k}$ is the randomly chosen bee, towards a direction of $j$ where the randomly selected bee $k \neq i$, which is subtracted from the same direction of taken $i$ th bee. The difference is then multiplied by the random number $\left(\phi_{i}^{j}\right)$ which varies within $[-1,1]$. Finally, this solution is added to the $j$ th vector of $S_{i}$ to get a $j$ th dimension of a new food position $A_{i}^{j}$, which means the updated solution of the current $S_{i}^{j}$. As shown in Figure 2, it is demonstrated that all other dimensions of $A_{i}^{j}$ are the same as those of $S_{i}$ and are generated in the neighborhood of $S_{i}^{j}$.

4.2.3. Onlooker Bees Phase. The procedure of onlooker bee phase comes just after finishing the critical role of the employed bee phase in ABC algorithm. During the procedure, all employed bees share the quality-wise information of the updated solutions and also the position information with the onlooker bees. After getting the information from the employed bees, the onlooker bees analyze the available information and select the promising candidate solutions probabilistically based on the fitness information with its fitness function. The probability prob $_{i}$ is calculated using the following expressions in

$$
\begin{aligned}
\text { fitness }_{i} & = \begin{cases}\frac{1}{F_{i}} & \text { if } F_{i} \geq 0 \\
1+\operatorname{abs}\left(F_{i}\right) & \text { if } F_{i}<0\end{cases} \\
\text { prob }_{i} & =\frac{\text { fitness }_{i}}{\sum_{i}^{P / 2} \text { fitness }_{i}},
\end{aligned}
$$

where $F_{i}$ is the objective function explained in Section 4.4 in (14) and $P$ is a population size mentioned in simulation parameters in Table 1.

4.2.4. Scout Bees Phase. In the scout bees phase, the employed bees are those whose fitness value of food source is not updated for a predetermined number of cycles. The food 


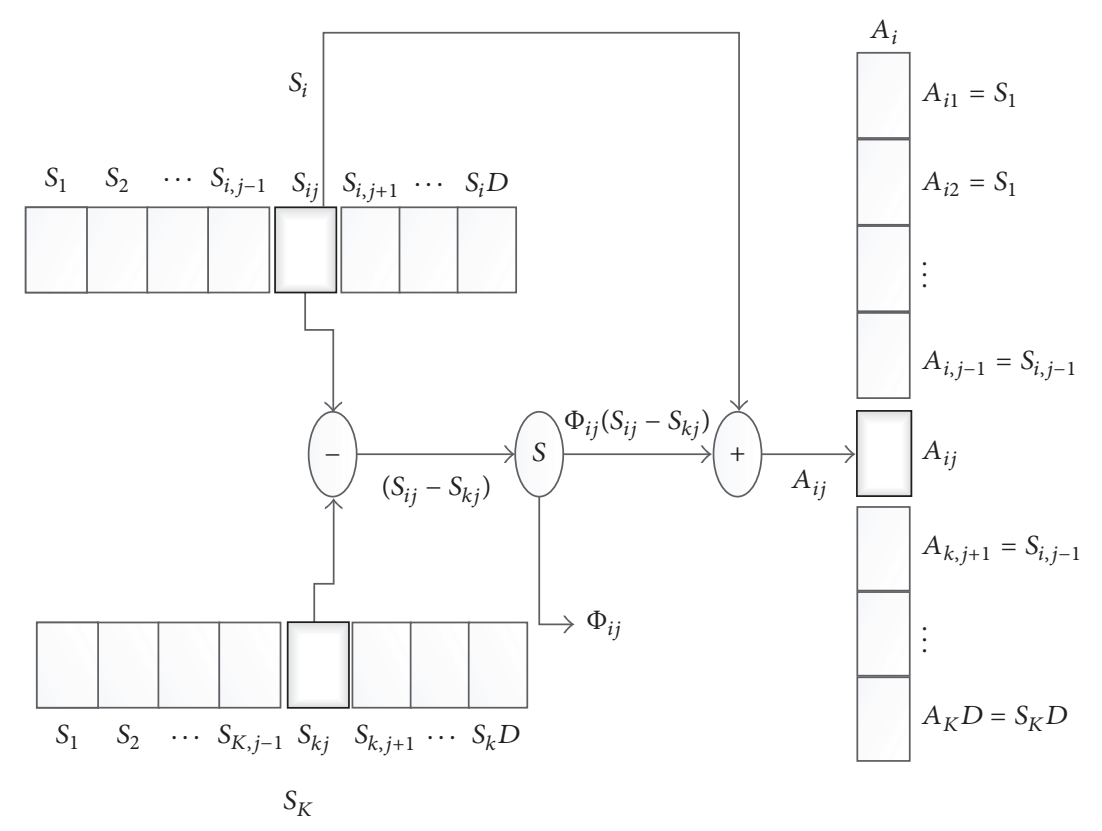

FIgURE 2: An example of the base station's position update.

TABLE 1: Simulation parameters.

\begin{tabular}{lc}
\hline$C_{f}$ & $15 \mathrm{GHz}$ \\
$P$ & 100 \\
$\lambda$ & 50 \\
$G$ & 200 \\
$P_{t}$ & $0.1-10 \mathrm{~W}$ \\
$A_{g}$ & $18 \mathrm{dBi}$ \\
$M_{g}$ & $2 \mathrm{~dB}$ \\
$C_{l}$ & $2 \mathrm{~dB}$ \\
$\mathrm{MNC}$ & 3000 \\
$N_{f}$ & $2 \mathrm{~dB}$ \\
$L_{b}$ & $2 \mathrm{~dB}$ \\
$K$ & 100 \\
$\mathrm{LG}_{\mathrm{ul} X}$ & -100 \\
$\mathrm{LT}_{\mathrm{ul} Y}$ & 100 \\
$\mathrm{LG}_{\mathrm{lr} X}$ & 100 \\
$\mathrm{LT}_{\mathrm{lr} Y}$ & -100 \\
$\phi$ & rand $[0$ to 1$]$ \\
$\mathrm{NU}$ & 10000 \\
\hline
\end{tabular}

source is assumed to be abandoned, and the scout bees phase starts by replacing their old solutions with searching for new solutions randomly according to (5) within the search space. For instance, if the $i$ th solution is abandoned, a new solution is generated to replace the original one using (5), where we set $i=1$. The predetermined number of cycles is a central control parameter which is also named a limit for abandonment. Assume that, for the BS, source $S_{i}$ is available in the search space; then the scout bees replace the old $S_{i}$ with a new $S_{i}$ food source.
4.3. Modified Artificial Bee Colony. The original idea of ABC algorithm performs a hierarchical optimization having a significant drawback in that it considers solutions over generations equally. This inherent disadvantage comes with the most of the population-based stochastic algorithms which relate to a premature convergence or stagnation towards a generation. When $\mathrm{ABC}$ tries to solve a complex problem having a large number of variables, the problem is a significant influence on the efficiency and accuracy of $\mathrm{ABC}[24,25]$. Therefore, the Modified ABC should be able to overcome the issue of the traditional $\mathrm{ABC}$. In our targeted $5 \mathrm{G}$ network planning problem, every variable relates to its neighborhood variables. Thus, if the value of one variable changes, it directly affects its neighborhood variables and indirectly the other individual variables. To improve the conventional $\mathrm{ABC}$ for getting an optimal solution for our problem is required. Therefore, ABC should be made more efficient.

The position updates in the conventional ABC applying (7) cannot make a large difference from the initialization of the population. After some iterations, all potential solutions work within a small proximity. In this issue, $\left(S_{i}^{j}-S_{k}^{j}\right)$, where $i$ and $k$ make a slight difference without improving, sometimes becomes negligible towards a generation. This phenomenon is called a premature convergence or stagnation if the globally optimal solution is not present in this small proximity. From this point of view, this conventional $\mathrm{ABC}$ is not an efficient algorithm according to [26]. Thus, for making convergence rate greater while applying standard $\mathrm{ABC}$ to constrained problems, we need to analyze the effect of the perturbation rate which can control the frequency of parameter changes. For controlling the parameters to determine the Scaling Factor (step size), an approach to improve the standard ABC in order to make convergence rate efficient is proposed. 
In the traditional version of $\mathrm{ABC}$, while producing a new solution $A_{i}$, it changes only one parameter of the parent solution $S_{i}$ which results in a slow convergence rate. To reduce this obstacle of the ABC optimization methods, the first change was done by the MABC as follows: our MABC proposes a new control parameter called modified mutation rate $\partial$. Here, for each $S_{i}^{j}$, a uniformly distributed random number $\Re_{i}^{j}$ between 0 and 1 is generated. If the random number $\Re_{i}^{j}$ is less than $\partial$, parameter $S_{i}^{j}$ modifies the following:

$$
A_{i}^{j}= \begin{cases}S_{i}^{j}+\phi_{i}^{j}\left(S_{i}^{j}-S_{k}^{j}\right), & \text { if } \mathfrak{R}_{i}^{j}<\partial, \\ S_{i}^{j}, & \text { otherwise }\end{cases}
$$

where $k \in\{1,2, \ldots, P\}, i \in\{1,2, \ldots, P\}$, and $j \in\{1,2, \ldots, D\}$ have randomly chosen indices. Here $k$ must have a different random number from $i$ and $\partial$ is the modified mutation rate which takes a value between 0 and 1 . If $\partial$ gets a lower value, the solution improves slowly, but while getting a higher one, it becomes a cause of greater diversity in an optimal solution and hence in the population. Additionally, the ratio of the variance operator is also modified in MABC algorithm. In the traditional $\mathrm{ABC}$, taking a random perturbation avoids getting stuck at local minima which add to the current solution in order to produce a new solution.

A different random number of the solutions in $S_{i}$ and $S_{k}$ where $k \neq i$ is weighted by a real random number called $\phi_{i}^{j} . \phi_{i}^{j}$ varies in the range $[-1,1]$, which is called a random perturbation of traditional $\mathrm{ABC}$. In our MABC, the solutions $S_{i}$ and $S_{k}$ vary within the range $[-\sigma, \sigma]$; hence, the second improvement of MABC was done by presenting the control parameter $(\sigma)$ as a Scaling Factor that means a step size to control the magnitude of the perturbation. A smaller value of $\sigma$ allows for the process in small steps, leading to slow convergence while having a larger value of $\sigma$ speeds up the steps, but it reduces the exploitation capability of the perturbation method. The function of $\sigma$ in (10) defines a heuristic rule which assigns different values depending on the number of generations. The mutation step size $\sigma(g)$ is given as a function for $g$ in (10). The employed bees and onlooker bees both use this expression to search for the neighbor food source. We have more enhancement in the algorithm regarding the fitness function evaluation which is counted as the third improvement for our MABC. If the number of fitness evaluations decreases, the algorithm runs faster than having more fitness evaluations. In our modified $\mathrm{MABC}$, what we have done differently for the traditional $\mathrm{ABC}$ is described in Algorithm 1 of modified employed bees and Algorithm 2 of modified onlooker bees. As we have used this expression in employed bees and onlooker bees during the process of these bees, the MABC algorithm is set only to evaluate those chromosomes which are already modified in the greedy selection method. If we apply this expression for selecting the neighbor food source, it does not always repeat a new food source position due to the constraints given in algorithms. It means that the MABC algorithm checks whether a food source has been modified or not before proceeding with the fitness function evaluation. This checking-in MABC helps to eliminate a number of fitness evaluations for the modified individuals that have already been evaluated in the past generation. We also considered that the algorithm can converge to an optimal solution.

$$
\sigma(g)=1-0.9 \times \frac{g}{G},
$$

where $G$ is a maximum generation number and current generation number $(g)$ varies from 0 to $G$. The mutation step size $\sigma(g)$ follows all variables of each vector in the population. In the start, it will decrease slowly from 1 at the beginning of the run during $(g=0)$ to 0.1 as the number of generations $g$ approaches $G$. Thus, this decrease of $\sigma(g)$ performs the best tuning capability of the proposed algorithm.

The fourth innovative point of our MABC is that it solves the problem of exploitation and the also convergence speed has a better tuning capability than the traditional $\mathrm{ABC}$. After getting a different random perturbation of $S_{i}$ and $S_{k}$, the proposed MABC calculates a neighborhood solution $\forall_{i}^{j}$ introduced in (11) using an inertia weight given in (12):

$$
\begin{aligned}
\forall_{i}^{j} & =S_{i}^{j} \times w+2\left(\psi_{i}^{j}-0.5\right)\left(S_{i}^{j}-S_{k}^{j}\right)+\phi_{i}^{j}\left(B_{i}^{j}-S_{k}^{j}\right), \\
w & =0.5+\frac{\operatorname{rand}()}{2},
\end{aligned}
$$

where $w$ is a random inertia weight which controls the impact of the previous solution $S_{i}^{j}$, the best-so-far solution is represented as $B_{i}^{j}$ of $j$ th dimension, $\phi_{i}^{j}$ is a random number within [0 to 1], and $\psi_{i}^{j}$ follows a mutation step size process instead of varying in the range of $[-1,1]$. The modified employed bees phase is described in Algorithm 1.

MABC uses (11) instead of calculating the neighborhood solution of conventional $\mathrm{ABC}$ in (7) in order to proceed with a better result in the employed bee phase. After completing the employed bee phase in our proposed MABC, the onlooker bees calculate a probability in (13) as follows:

$$
\operatorname{prob}_{i}=\frac{0.9 \times F_{i}}{\max (F)}+0.1,
$$

where $F_{i}$ is the fitness value of the $i$ th solution in the population. The modified onlooker bees phase is described in Algorithm 2. The rest of all the procedures after the onlooker bees and scout bees phases follow the same steps as conventional $\mathrm{ABC}$. In addition to these innovative points mentioned above, a new control parameter $\partial$ (called modified mutation rate) and a step size $\sigma$ both produce a greater diversity to an optimal solution and eliminate a number of fitness evaluations for modified individuals that have already been evaluated in the past generation. As a result, they solve the problem of exploitation with the convergence speed having a better tuning capability. Therefore, our MABC has so far obtained better results than the standard $\mathrm{ABC}$. The overall procedure of our proposed MABC is described in Algorithm 3.

4.4. Fitness Evaluation. A fitness function uses a type of objective function in EAs which helps to get a solution from 


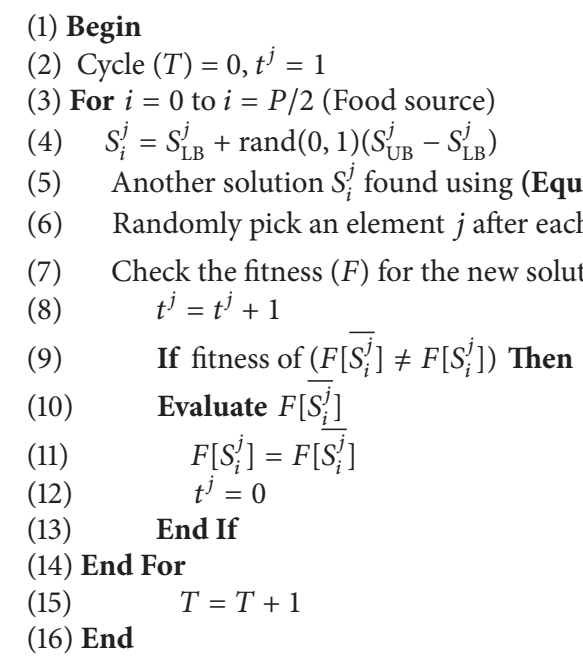

Algorithm 1: The modified employed bees.

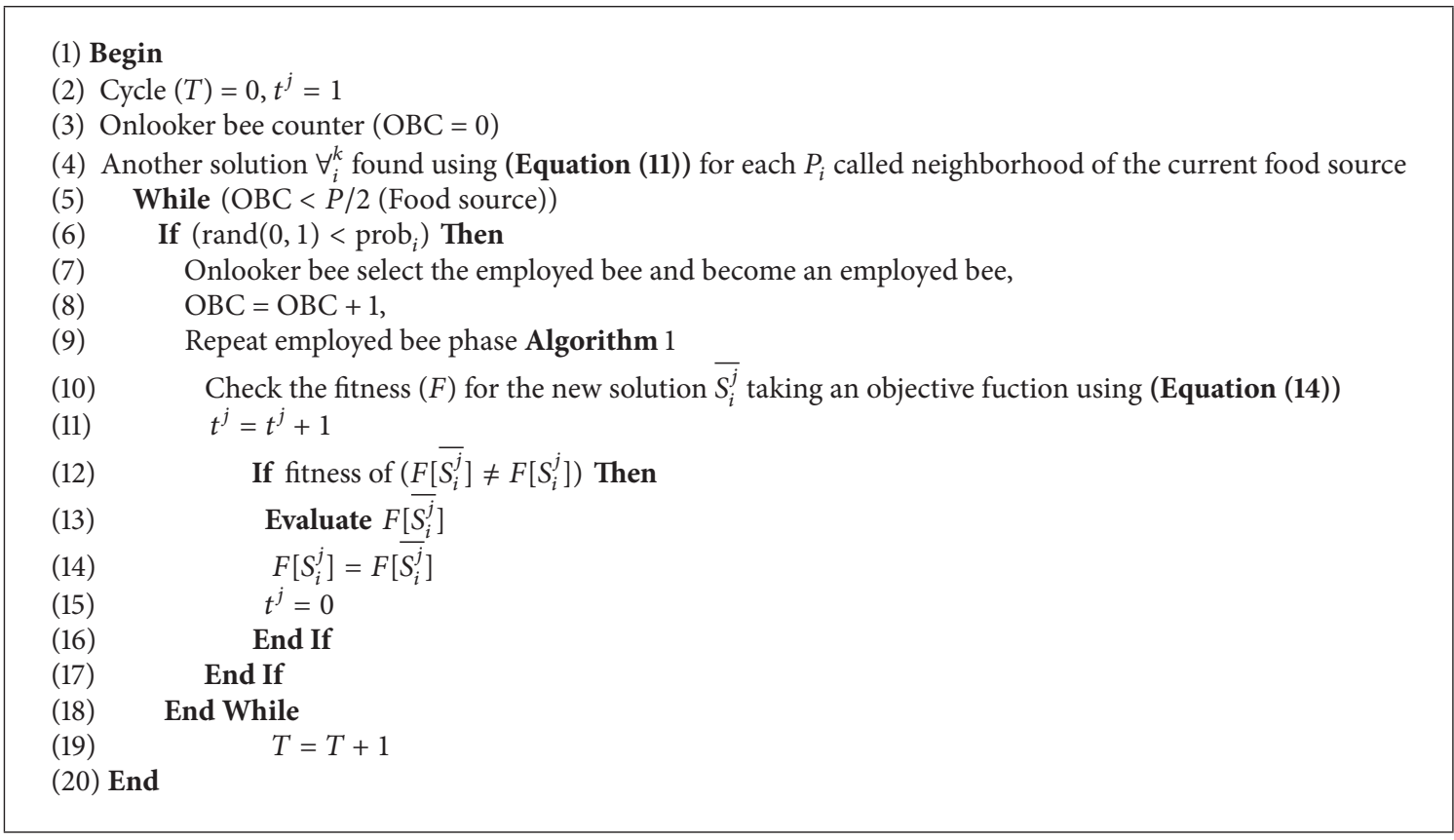

Algorithm 2: The modified onlooker bees.

the evaluation of each for the survival of next generation. In $\mathrm{ABC}$, each single solution exists in the target search space, which we call the type of bees. All of the bees have fitness value which is evaluated by the taken objective function to be optimized. Our approach is formulated by using the objective function given in (14) for getting the fitness $\left(F_{i}\right)$ of the optimal network configuration as follows:

$$
F_{i}=\left[\frac{\mathrm{UE}^{2}}{T_{P} \times \text { ActiveBSes }^{2}}\right],
$$

where UE is the number of connected users to the BSes, $T_{P}$ represents the total transmit power, and ActiveBSes represent the number of BS such that $T_{P}$ is configured for each user to be connected to a BS. We defined the maximum number of generations $G$ as for the termination criteria. The proposed algorithm terminates after executing simulation $T_{g}$ generations and returns the best-so-far solution.

\section{Computational Complexity}

In this section, we discuss the complexity of our proposed $\mathrm{MABC}$ algorithm. The proposed algorithm is described in the following five parts: (i) the initialization of the food source, (ii) the search operation of modified employed bees, (iii) 


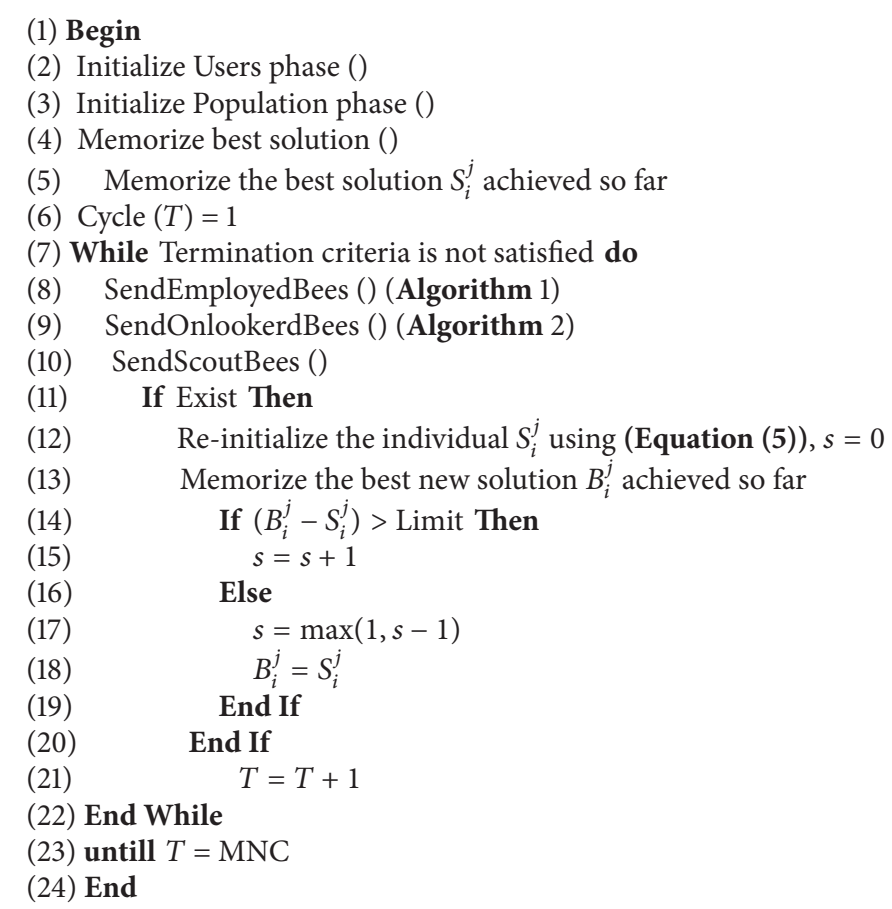

Algorithm 3: Pseudocode of MABC.

the probability of food sources, (iv) the search operation of onlooker bees, and (v) the search operation of scouts bees. First, the computational complexity of the initialization is $O(F K D)$ where $F$ is the food number that is equal to the half of the colony size; $K$ is the number of base stations (BSes); $D$ is the vector dimension. Second, the complexity of the search operation of employed bees is $O\left(F K D+F K D N_{u}\right)$ where $N_{u}$ is the number of users. Third, the complexity of food source's probability is $O(F K)$. Fourth, the complexity of search operation of onlooker bees is $O\left(F K D+F K D N_{u}\right)$. Last, the complexity of search operation of scout bees is $O(F K D)$. Therefore, the overall computational complexity of our proposed scheme is $O\left(\left(1 / N_{f} e\right)\left(F K D+\lambda\left(F K D N_{u}+F K+D\right)\right)\right)$ where $N_{f} e$ is number of fitness evaluations; $\lambda$ is the number of iterations. Now we analyze the time complexity of the original $\mathrm{ABC}$ algorithm. The total time complexity of the traditional $\mathrm{ABC}$ is $\mathrm{O}\left(F K D+\lambda\left(2 F K D N_{u}+2 F K+D\right)\right)$ [27]. This original $\mathrm{ABC}$ algorithm has more than one fitness evaluation for each individual during the generation. Each employed bee tests a neighbor food source for their quality based on the fitness function. It means that the fitness function evaluates double for all the individuals during the search operations through these bees. Compared to the traditional ABC algorithm, our approach $\mathrm{MABC}$ does not add any extra operations regarding the complexity effect. Even MABC does not run the fitness function evaluation for all the individuals twice during the search process of employed bees and onlooker bees if they already found better nectar food source at the first time of the evaluation. With these constraints, our MABC has a better fitness value than original $\mathrm{ABC}$ and other EAs without losing the good performance as mentioned in Table 6. However, this operation can help MABC to run faster than $\mathrm{ABC}$. If it does not happen, then, in the worst case, our MABC and the original $\mathrm{ABC}$ keep the same computational complexity. For RCGA, its total time complexity is $O\left(P K+G\left(P^{2}+P K^{2}+P\left(N_{u}+\right.\right.\right.$ $\left.K N_{u}\right)$ )) where $P$ is the population size; $G$ is the maximum number of generations [28]. The complexity of the MRGA is the same as that of RCGA because of having same operations used in MRGA except a difference between the operation of crossover and mutation [4]. These operations do not affect the computational complexity between RCGA and MRGA. For DE, its computational complexity is $\mathrm{O}\left(P K+P K N_{u}+\right.$ $\left.G P\left(N_{u}+K N_{u}\right)\right)$ where all the notations are described above [29]. Therefore, MABC has the same complexity as that of the original ABC. MRGA also has the same complexity as that of RCGA because of no additional operations used in the algorithm regarding complexity. DE has less computational complexity than MRGA and RCGA, but it has the same complexity as both our MABC and the original ABC.

\section{Results and Discussion}

In this section, we represent some numerical results obtained from the application of Evolutionary Algorithms such as DE, RGA, MRGA, ABC, and MABC. The performance evaluation of our proposed Modified ABC is performed with these algorithms in a fair manner. The aim of these experiments does show not only the effectiveness of our algorithm on realistic network planning but also the impact that energy consumption issues have pointed out in our simulation. Firstly, the modified algorithm is evaluated by concerning the best-optimized power level and its location problem for 


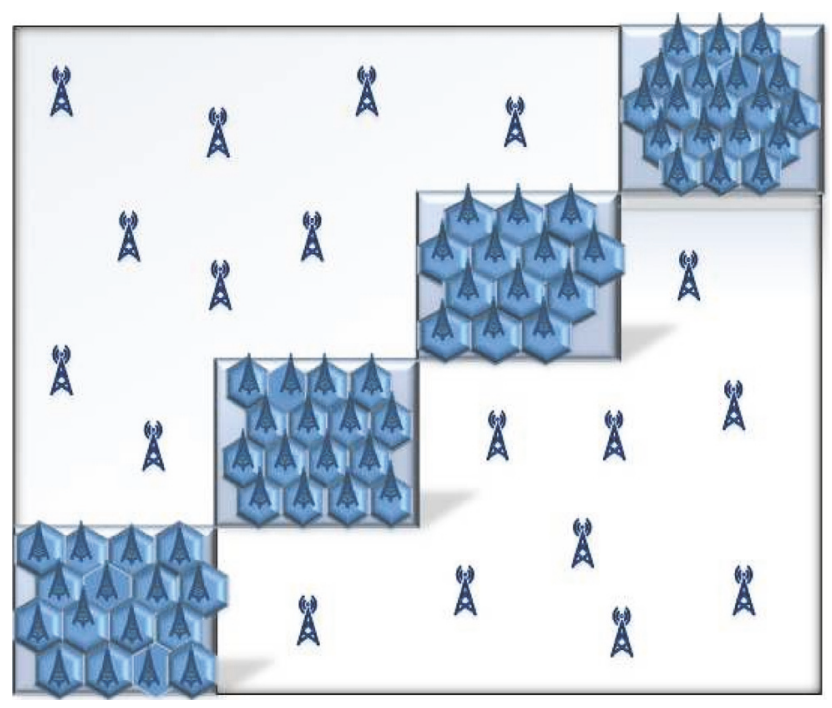

Figure 3: Simulation environment.

5G BSes with the standard ABC, RGA, and DE. In this scenario, we obtained our experiment results regarding the number of active BSes and transmission power as an energy consumption with the connected users in comparison with conventional DE, RGA, and ABC.

Simulation parameters are considered in Table 1. In this table, we have taken some of the constant variables such as carrier frequency $\left(C_{f}\right)$, FDD frame structure, receiver antenna gain $\left(A_{g}\right)$, bandwidth, MHA gain $\left(M_{q}\right)$, cable loss $\left(C_{l}\right)$, noise figure $\left(N_{f}\right)$, and body loss $\left(L_{b}\right)$. Our decision variables are defined as a population size $(P)$, maximum number of iterations $(\lambda)$, transmission power $(Q)$, and so forth. In our operations, the environment area is assumed to represent $(X, Y)$ as $(-100.00,100.00)$ and $(100.00,-100.00)$ in meter where BSes and UEs are considered to be connected in the given area of interest. The users are supposed to be allocated as an specific point by using their accuracy range in the given area as this takes a new feature in $5 \mathrm{G}$ wireless networks and for the future generation, too. The possible users connect to those BSes which are active for servicing the best quality based on the network planning. We have performed our experiment and reported values to estimate the best location for $5 \mathrm{G}$ base stations. The proposed simulation environment has been shown in Figure 3, where hexagon boxes represent centers with an entirely covered area with users occupied in an urban area. The circle shape represents an area which is allocated by an optimum BS in our proposed and the standard algorithms such as ABC, RGA, and DE. All useful notations are used in our paper mentioned in "Notations."

Our simulation results are calculated with over 50 independent runs. The comparison terms are taken for the modified RGA with Box Crossover Rate $(\mathrm{BCR})=0.1$ and Mutation Rate $\left(M_{r}\right)=0.2$. For standard DE, the Scaling Factor $(\mathrm{SF})=0.5$ and Crossover Rate $(\mathrm{CR})=0.9$ are used. For standard RGA, $M_{r}=0.2$ is used. The proposed MABC takes mutation rates with their step size as described in Section 6.

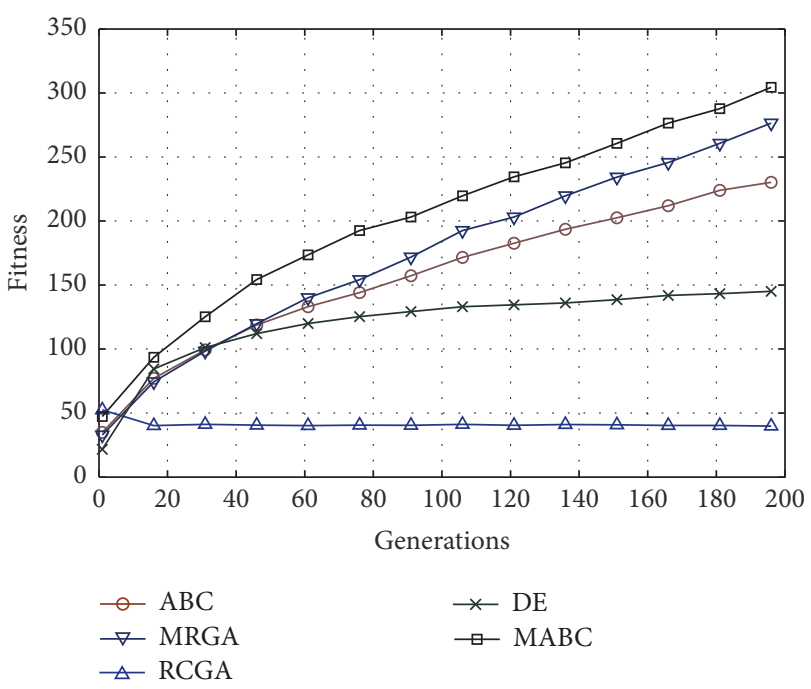

FIGURE 4: Convergence graphs.

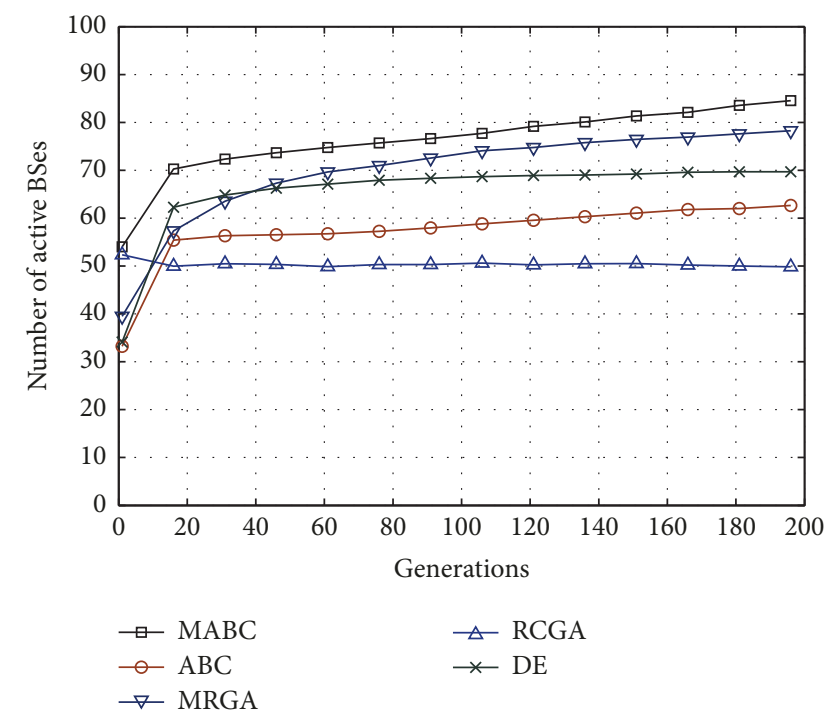

FIgURE 5: The number of active base stations towards a generation.

The convergence graph has been shown in Figure 4 where MABC performs better towards the upcoming generations than the MRGA and all other application of Evolutionary Algorithms such as the standard $\mathrm{ABC}, \mathrm{RGA}$, and $\mathrm{DE}$. While comparing our modified algorithms with these existing algorithms, we found that the standard RGA gets lightly equal and even worse fitness value towards a generation because the shuffling happens again and again by using Box Crossover which is used in RGA algorithms. The modified RGA gets better fitness than RGA and DE. This is because they are not changing their chromosome every time in crossover operation. RGA has better fitness value than the standard RGA, $A B C$, and DE but is not better than our MABC as we have performed the modification in their bees of standard $A B C$ which give better fitness value than that of the standard $\mathrm{ABC}, \mathrm{RGA}$, and $\mathrm{DE}$ for the best network planning in $5 \mathrm{G}$ networks.

Figure 5 shows the number of active BSes with the standard ABC, RCGA, DE and our MRGA, and MABC. We 


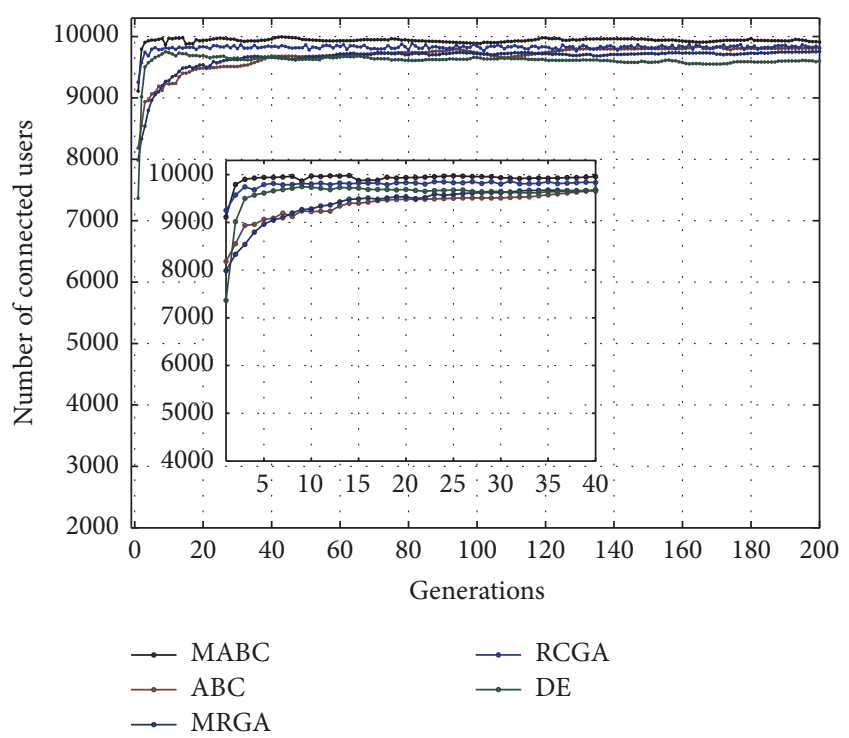

FIGURE 6: The number of connected users towards a generation.

can see that MABC can have more active BSes than even MRGA [4] and the standard ABC, RCGA, and DE. The EAs such as DE, RCGA, and ABC hold less activated BSes and serve less users at the same time in comparison with the MRGA and ABC. The results lead to less fitness value as it depends on the fitness function. The randomness of the EAs produces more chances for the network operator to find better BS combinations. However, the MABC and MRGA keep more active base stations than the standard ABC, RCGA, and DE which uses the advantage of the higher computational complexity that depends on their level of crossover and mutation of RCGA and DE. For ABC, it depends on the standard role of bees. We emphasize that standard RCGA and DE have performed well in terms of the less active base stations but serve users insufficiently and also could not perform well regarding their power consumption. That is why the MRGA and MABC increase the number of active base stations more with less power consumption than the standard ABC, RCGA, and DE to achieve better fitness.

Figure 6 shows a number of connected users towards a generation with MABC, MRGA, and the standard $A B C$, RCGA, and DE. As this figure shows, that all of the algorithms performed well regarding the coverage area by users with the connection of their active base stations. But there is still a difference in performance after reaching the 40th generation to provide excellent coverage in our simulation environment among these standard and modified algorithms. Figure 6 showed the difference is how these algorithms are performing slightly different from the starting generation till 40th generation.

The performance of the transmission power consumption among the proposed MABC, MRGA, and traditional ABC, RCGA, and DE has been shown in Figure 7. Our proposed MABC has performed well for consuming less power than the other algorithms [4]. According to the generation, this statement is true because the power is almost constant in

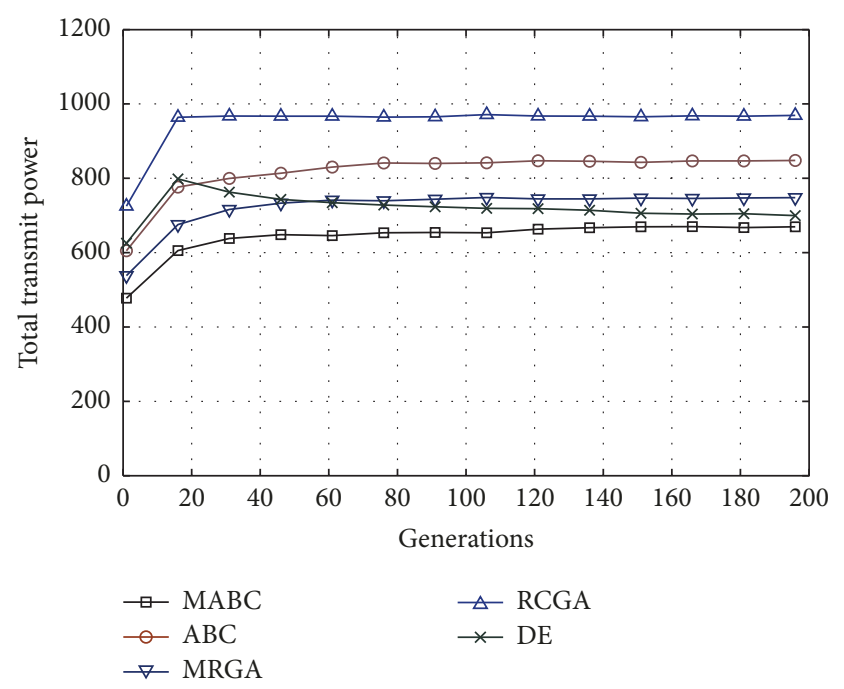

FIGURE 7: The total transmitting power towards a generation.

TABLE 2: The $t$-value for MABC and ABC in 49 degrees of freedom is significant at a QoS level of significance by two-tailed $t$-test.

\begin{tabular}{lccc}
\hline & The modified ABC & ABC & $t$-value \\
\hline Average & 308.68278 & 145.654808 & $\mathbf{2 . 0 0 0 8 6 E}-\mathbf{0 7}$ \\
Stdev & 77.82408737 & 15.8800909196 & \\
\hline
\end{tabular}

most of the scheme except for DE. As we see in Figure 7, the performance of DE and MRGA [4] was quite similar after reaching over the 60th generation and later DE performed well in comparison to MRGA. In the starting generation, we can see that MRGA has less total power consumption than all of three mechanisms named ABC, MRGA, and RCGA except $\mathrm{MABC}$ but while going to the next generation, its consumption goes high because of the shuffling of their chromosome during the reproduction by crossover. According to $\mathrm{ABC}$ performance, the traditional $\mathrm{ABC}$ could not perform better than MRGA and DE but have performed better than RCGA. Thus we have modified the traditional $\mathrm{ABC}$ as MABC where we achieved very less power consumption from the initial point of the generation because of modifying their mutation step size on their bee phases. By making traditional ABC as a novel ABC after modifying, we got the good results regarding the power consumption. The statistical results have been tabulated in Tables 2-5, respectively. Firstly, Table 2 shows the performance of our proposed MABC and the traditional ABC where we get $t$-value $2.00086 E-07$. Table 3 shows the performance of MRGA where we are getting $t$-value 0.023381 . Table 4 shows the comparison of our MABC and RCGA where we get $t$-value $4.03195 E-29$. Lastly, the results of our proposed MABC are shown, compared with the traditional $\mathrm{DE}$ where $t$-value gives $4.05318 E-20$ in Table 5 . Hence this proves that the MABC is statistically better than the traditional ABC, RCGA, and DE. 
TABLE 3: The $t$-value for MABC and MRGA in 49 degrees of freedom is significant at a QoS level of significance by two-tailed $t$-test.

\begin{tabular}{lccc}
\hline & The modified ABC & The modified RGA & $t$-value \\
\hline Average & 308.68278 & 277.270614 & $\mathbf{0 . 0 2 3 3 8 1}$ \\
Stdev & 77.82408737 & 56.70863407 & $\mathbf{0 . 0 2 3 3}$ \\
\hline
\end{tabular}

TABLE 4: The $t$-value for MABC and RCGA in 49 degrees of freedom is significant at a QoS level of significance by two-tailed $t$-test.

\begin{tabular}{lccc}
\hline & The modified ABC & RCGA & $t$-value \\
\hline Average & 308.68278 & 39.76453 & $\mathbf{4 . 0 3 1 9 5 E}$ - 29 \\
Stdev & 77.82408737 & 2.399219965 & \\
\hline
\end{tabular}

TABLE 5: The $t$-value for MABC and DE in 49 degrees of freedom is significant at a QoS level of significance by two-tailed $t$-test.

\begin{tabular}{lccc}
\hline & The Modified ABC & DE & $t$-value \\
\hline Average & 308.68278 & 145.654808 & $\mathbf{4 . 0 5 3 1 8 E}-\mathbf{2 0}$ \\
Stdev & 77.82408737 & 15.8800909 & \\
\hline
\end{tabular}

\section{Conclusion}

In this paper, we formulate a network planning optimization problem with our proposed Modified ABC (MABC) algorithm, the standard ABC, RCGA, and DE. The key objective of this network planning problem is to minimize the power consumption while using the minimum number of active base stations with their connected users in order to assure a certain quality of service to the users. Since this optimization problem is an NP-hard problem, it consumes tremendous resources such as computation time and requires the evaluation of a number of expensive fitness functions for a high-quality solution using the application of Evolutionary Algorithms (EAs). Therefore, the insight of the EAs has a better tradeoff between resources and the quality of solutions. The application of EAs is an intelligent tool which provides us with an optimum high-quality solution to the optimization problems with a huge search space. We have compared the three legacy algorithms (i.e., ABC, RCGA, and DE) with our MABC in performance evaluation. The MABC has successfully found much better configuration by comparing with the conventional DE and RCGA and even the modified RCGA (MRGA) in order to locate a proper location and also to adjust the range of the power along with their connected users. Experimental results classified the application of EAs regarding the performance and the number of function evaluations. This indicates that our MABC can guide us towards choosing an efficient way to achieve high transmit power saving and to satisfy coverage constraints for 5G wireless networks. As for future work, we will enhance our MABC algorithm for handover scenarios (e.g., vehicular networks) where UEs are moving fast in the $5 \mathrm{G}$ wireless networks.

\section{Notations}

\section{Parameters}

$P_{t}: \quad$ Transmission power

$C_{l}: \quad$ Cable loss

$A_{g}: \quad$ Receiver antenna gain

$N_{f}: \quad$ Noise figure

$L_{b}$ : Body loss

$C_{f}$ : Carrier frequency

$G$ : $\quad$ Maximum number of generations

$\lambda: \quad$ Maximum number of iterations

NU: Number of users

$F$ : Objective function

$F_{i}: \quad$ Fitness value of the $i$ th solution

SF: $\quad$ Scaling Factor for RCGA and MRGA

$M_{r}$ : Mutation Rate for RCGA and MRGA

BCR: Box Crossover Rate

$P$ : $\quad$ Population size

$\sigma(g)$ : Mutation step size varying with current generation $g$

$w: \quad$ Random inertia weight

$\phi: \quad$ Random number

MNC: Maximum number of cycles

$\mathrm{LG}_{\mathrm{ulX}}$ : Longitude (upper-left $X$ )

$\mathrm{LT}_{\mathrm{ul} Y}$ : Latitude (upper-left $Y$ )

$\mathrm{LG}_{\mathrm{lr} X}$ : Longitude (lower-right $X$ )

$\mathrm{LT}_{\operatorname{lr} Y}$ : Latitude (lower-right $Y$ ).

\section{Abbreviations}

EAs: Evolutionary Algorithms

GA: Genetic Algorithm

RCGA: Real-Coded Genetic Algorithm

MRGA: Modified RGA

DE: Differential Evolution

ABC: Artificial Bee Colony

MABC: Modified ABC.

\section{Conflicts of Interest}

The authors declare that there are no conflicts of interest regarding the publication of this paper.

\section{Acknowledgments}

This research was supported by Institute for Information \& Communications Technology Promotion (IITP) grant funded by the Korean Government (MSIP) (no. B0717-170070) and also by Basic Science Research Program through 
TABLE 6: Comparison of results obtained for their fitness value by MABC, ABC, MRGA, RCGA, and DE with 50 test numbers getting in 200th-generation time.

\begin{tabular}{|c|c|c|c|c|c|c|c|c|}
\hline \multicolumn{9}{|c|}{ Fitness value } \\
\hline Iteration & MABC & $\mathrm{ABC}$ & MABC & MRGA & MABC & RCGA & MABC & $\mathrm{DE}$ \\
\hline$\overline{1}$ & 336.569 & 220.5592 & 336.569 & 288.199 & 336.569 & 38.4158 & 336.569 & 122.4345 \\
\hline 2 & 326.7795 & 232.8575 & 326.7795 & 256.7098 & 326.7795 & 38.138 & 326.7795 & 122.4345 \\
\hline 3 & 136.3359 & 178.0451 & 136.3359 & 184.0869 & 136.3359 & 38.9982 & 136.3359 & 147.3075 \\
\hline 4 & 478.0451 & 263.1525 & 478.0451 & 263.0525 & 478.0451 & 37.4129 & 478.0451 & 147.3413 \\
\hline 5 & 363.8525 & 206.3958 & 363.8525 & 243.324 & 363.8525 & 38.8615 & 363.8525 & 157.4405 \\
\hline 6 & 399.3313 & 229.994 & 399.3313 & 266.0057 & 399.3313 & 38.9466 & 399.3313 & 168.3165 \\
\hline 7 & 497.6723 & 343.9293 & 497.6723 & 343.9293 & 497.6723 & 38.2377 & 497.6723 & 182.6838 \\
\hline 8 & 343.9293 & 279.6975 & 343.9293 & 217.4483 & 343.9293 & 39.747 & 343.9293 & 138.1339 \\
\hline 9 & 413.9853 & 217.6088 & 413.9853 & 257.755 & 413.9853 & 38.792 & 413.9853 & 137.7067 \\
\hline 10 & 221.5153 & 243.5444 & 221.5153 & 270.0002 & 221.5153 & 41.7116 & 221.5153 & 133.2046 \\
\hline 11 & 320.0002 & 217.1254 & 320.0002 & 240.9877 & 320.0002 & 40.4955 & 320.0002 & 133.0904 \\
\hline 12 & 317.9254 & 223.4749 & 317.9254 & 282.6575 & 317.9254 & 42.9836 & 317.9254 & 144.4086 \\
\hline 13 & 382.6575 & 223.1256 & 382.6575 & 282.9843 & 382.6575 & 45.9056 & 382.6575 & 150.9968 \\
\hline 14 & 223.1256 & 216.3692 & 223.1256 & 306.8127 & 223.1256 & 44.3962 & 223.1256 & 139.5661 \\
\hline 15 & 270.1614 & 245.5956 & 270.1614 & 313.6375 & 270.1614 & 38.0587 & 270.1614 & 137.1016 \\
\hline 16 & 254.719 & 185.7675 & 254.719 & 204.5175 & 254.719 & 39.0704 & 254.719 & 176.6061 \\
\hline 17 & 287.0222 & 205.4735 & 287.0222 & 240.775 & 287.0222 & 41.1525 & 287.0222 & 150.9161 \\
\hline 18 & 321.2165 & 178.1283 & 321.2165 & 185.5978 & 321.2165 & 41.6386 & 321.2165 & 131.9684 \\
\hline 19 & 378.1283 & 247.4768 & 378.1283 & 265.8708 & 378.1283 & 36.8584 & 378.1283 & 132.8054 \\
\hline 20 & 247.9118 & 267.8852 & 247.9118 & 310.4483 & 247.9118 & 44.0629 & 247.9118 & 162.799 \\
\hline 21 & 297.8852 & 215.574 & 297.8852 & 261.19 & 297.8852 & 35.4141 & 297.8852 & 172.6942 \\
\hline 22 & 220.0042 & 210.3367 & 220.0042 & 309.0168 & 220.0042 & 40.118 & 220.0042 & 125.2206 \\
\hline 23 & 210.9367 & 190.0167 & 210.9367 & 235.3166 & 210.9367 & 43.9276 & 210.9367 & 142.237 \\
\hline 24 & 227.8001 & 197.3563 & 227.8001 & 204.8888 & 227.8001 & 37.6756 & 227.8001 & 160.4762 \\
\hline 25 & 197.3063 & 229.4653 & 197.3063 & 280.4655 & 197.3063 & 37.1763 & 197.3063 & 129.5167 \\
\hline 26 & 233.043 & 274.8593 & 233.043 & 301.5113 & 233.043 & 36.6548 & 233.043 & 132.8081 \\
\hline 27 & 301.5113 & 198.5159 & 301.5113 & 224.5502 & 301.5113 & 37.8516 & 301.5113 & 181.9633 \\
\hline 28 & 291.0059 & 187.1543 & 291.0059 & 237.3015 & 291.0059 & 37.8559 & 291.0059 & 128.0427 \\
\hline 29 & 321.4146 & 226.2556 & 321.4146 & 331.5433 & 321.4146 & 41.3885 & 321.4146 & 161.0378 \\
\hline 30 & 257.9592 & 265.8664 & 257.9592 & 265.8664 & 257.9592 & 40.9125 & 257.9592 & 135.5947 \\
\hline 31 & 365.8994 & 276.1566 & 365.8994 & 330.5423 & 365.8994 & 39.8699 & 365.8994 & 132.1572 \\
\hline 32 & 308.9495 & 242.4229 & 308.9495 & 289.2582 & 308.9495 & 39.6183 & 308.9495 & 143.372 \\
\hline 33 & 342.9229 & 229.6357 & 342.9229 & 287.8494 & 342.9229 & 42.3116 & 342.9229 & 158.5198 \\
\hline 34 & 262.8943 & 288.9151 & 262.8943 & 345.6238 & 262.8943 & 40.8357 & 262.8943 & 147.5854 \\
\hline 35 & 292.465 & 125.2196 & 292.465 & 197.748 & 292.465 & 38.8482 & 292.465 & 147.7685 \\
\hline 36 & 397.748 & 402.1246 & 397.748 & 452.8917 & 397.748 & 36.3569 & 397.748 & 136.2769 \\
\hline 37 & 402.1916 & 303.3515 & 402.1916 & 312.0491 & 402.1916 & 43.8927 & 402.1916 & 129.7897 \\
\hline 38 & 271.1631 & 283.7516 & 271.1631 & 313.1315 & 271.1631 & 37.1562 & 271.1631 & 149.7464 \\
\hline 39 & 213.1315 & 202.5987 & 213.1315 & 259.6116 & 213.1315 & 39.0499 & 213.1315 & 164.2077 \\
\hline 40 & 202.5987 & 262.1589 & 202.5987 & 291.0349 & 202.5987 & 40.5539 & 202.5987 & 134.8221 \\
\hline 41 & 230.1549 & 315.8854 & 230.1549 & 386.4251 & 230.1549 & 41.4009 & 230.1549 & 137.6344 \\
\hline 42 & 316.4251 & 163.4352 & 316.4251 & 191.4861 & 316.4251 & 39.7959 & 316.4251 & 145.1443 \\
\hline 43 & 323.0682 & 158.5565 & 323.0682 & 258.3565 & 323.0682 & 40.3688 & 323.0682 & 178.4081 \\
\hline 44 & 458.3565 & 270.9059 & 458.3565 & 343.0924 & 458.3565 & 39.3066 & 458.3565 & 133.0197 \\
\hline 45 & 289.0719 & 197.5141 & 289.0719 & 206.7901 & 289.0719 & 44.3265 & 289.0719 & 143.9841 \\
\hline 46 & 397.5341 & 256.4257 & 397.5341 & 389.7103 & 397.5341 & 39.494 & 397.5341 & 125.5549 \\
\hline 47 & 356.4256 & 169.1989 & 356.4256 & 228.2749 & 356.4256 & 39.791 & 356.4256 & 153.9786 \\
\hline 48 & 214.6505 & 233.8152 & 214.6505 & 248.5624 & 214.6505 & 38.7579 & 214.6505 & 156.4837 \\
\hline 49 & 333.8152 & 281.1153 & 333.8152 & 374.9021 & 333.8152 & 37.5592 & 333.8152 & 131.4135 \\
\hline \multirow[t]{3}{*}{50} & 374.9021 & 251.4721 & 374.9021 & 279.7401 & 374.9021 & 36.0738 & 374.9021 & 146.0198 \\
\hline & $t$-test & 0.03 & $t$-test & $4 E-29$ & $t$-test & $4.1 \mathrm{E}-20$ & $t$-test & $2 \mathrm{E}-07$ \\
\hline & $F$-test & 0.029 & $F$-test & $5.6 \mathrm{E}-61$ & $F$-test & $1.4 \mathrm{E}-21$ & $F$-test & 0.00188 \\
\hline
\end{tabular}


the National Research Foundation of Korea (NRF) funded by the Ministry of Education (no. 2017R1D1A1B03035885).

\section{References}

[1] S. U. Worldwide, retrieved from emarketer: http://www.emarketer .com/article/Smartphone-Users-Worldwide-Will-Total-175-Billion2014/1010536, 2014.

[2] H. Holma and A. Toskala, LTE for UMTS: OFDMA and SCFDMA Based Radio Access, John Wiley \& Sons, 2009.

[3] T. Han and N. Ansari, "Optimizing cell size for energy saving in cellular networks with hybrid energy supplies," in Proceedings of the IEEE Global Communications Conference (GLOBECOM '12), pp. 5189-5193, IEEE, Anaheim, Calif, USA, December 2012.

[4] R. Sachan, T. J. Choi, and C. W. Ahn, "A Genetic Algorithm with Location Intelligence Method for Energy Optimization in 5G Wireless Networks," Discrete Dynamics in Nature and Society, vol. 2016, Article ID 5348203, 2016.

[5] P. Kela, M. Costa, J. Salmi et al., "A novel radio frame structure for 5G dense outdoor radio access networks," in Proceedings of the 81st IEEE Vehicular Technology Conference, VTC Spring 2015, Glasgow, UK, May 2015.

[6] P. Pirinen, "A brief overview of 5G research activities," in Proceedings of the 1st IEEE International Conference on $5 G$ for Ubiquitous Connectivity (5GU '14), pp. 17-22, IEEE, Äkäslompolo, Finland, November 2014.

[7] R. Di Taranto, S. Muppirisetty, R. Raulefs, D. Slock, T. Svensson, and $\mathrm{H}$. Wymeersch, "Location-aware communications for $5 \mathrm{G}$ networks: how location information can improve scalability, latency, and robustness of 5G," IEEE Signal Processing Magazine, vol. 31, no. 6, pp. 102-112, 2014.

[8] S. Sakthivel and R. Suresh, "A genetic algorithm approach to solve mobile base station location problem," International Journal of Soft Computing, vol. 1, no. 3, pp. 160-165, 2006.

[9] V. Berrocal-Plaza, M. A. Vega-Rodríguez, J. A. Gómez-Pulido, and J. M. Sánchez-Pérez, "Artificial bee colony algorithm applied to WiMAX network planning problem," in Proceedings of the 11th International Conference on Intelligent Systems Design and Applications (ISDA '11), pp. 504-509, Córdoba, Spain, November 2011.

[10] S. Okasaka, S. Onoe, S. Yasuda, and A. Maebara, "A new location updating method for digital cellular systems," in Proceedings of the 41st IEEE Vehicular Technology Conference, pp. 345-350, May 1991.

[11] D. Plassmann, "Location management strategies for mobile cellular networks of 3rd generation," in Proceedings of the 1994 IEEE 44th Vehicular Technology Conference. Part 2 (of 3), pp. 649-653, June 1994.

[12] K. L. Yeung and T.-S. P. Yum, "Comparative study on location tracking strategies in cellular mobile radio systems," in Proceedings of the 1995 IEEE Global Telecommunications Conference. Part 2 (of 3), pp. 22-28, November 1995.

[13] A. Bar-Noy and I. Kessler, "Tracking Mobile Users in Wireless Communications Networks," IEEE Transactions on Information Theory, vol. 39, no. 6, pp. 1877-1886, 1993.

[14] H. M. Ali, S. Ashrafinia, J. Liu, and D. Lee, "Broadband wireless network planning using evolutionary algorithms," in Proceedings of the 2013 IEEE Congress on Evolutionary Computation, CEC 2013, pp. 1045-1052, Cancun, Mexico, June 2013.

[15] Y. Yu, S. Murphy, and L. Murphy, "Planning base station and relay station locations for IEEE 802.16 j network with capacity constraints," in Proceedings of the 2010 7th IEEE Consumer Communications and Networking Conference, CCNC 2010, pp. 405-409, Las Vegas, NV, USA, January 2010.

[16] E. Pinheiro, R. Bianchini, E. V. Carrera, and T. Heath, "Load balancing and unbalancing for power and performance in cluster-based systems," in Proceedings of the Workshop on Compilers and Operating Systems for Low Power, vol. 180, pp. 182-195, Barcelona, Spain, 2001.

[17] A. Chatzipapas, S. Alouf, and V. Mancuso, "On the minimization of power consumption in base stations using on/off power amplifiers," in Proceedings of the 1st IEEE Online Conference on Green Communications (GreenCom '11), pp. 18-23, IEEE, New York, NY, USA, September 2011.

[18] F. Boccardi, R. W. Heath, A. Lozano, T. L. Marzetta, and P. Popovski, "Five disruptive technology directions for 5G," IEEE Communications Magazine, vol. 52, no. 2, pp. 74-80, 2014.

[19] H. G. Myung, “Technical overview of 3GPP LTE,” Polytechnic University of New York, 2008.

[20] A. Al-Samawi, A. Sali, N. K. Noordin, M. Othman, and F. Hashim, "Base station location optimisation in LTE using genetic algorithm," in Proceedings of the International Conference on Information and Communication Technology Convergence (ICTC '13), pp. 336-341, IEEE, Jeju, Republic of Korea, October 2013.

[21] A. Ligeti and J. Zander, "Minimal cost coverage planning for single frequency networks," IEEE Transactions on Broadcasting, vol. 45, no. 1, pp. 78-87, 1999.

[22] B. Jalili and M. Dianati, "Application of taboo search and genetic algorithm in planning and optimization of UMTS radio networks," in Proceedings of the 6th International Wireless Communications and Mobile Computing Conference (IWCMC '10), pp. 143-147, ACM, New York, NY, USA, July 2010.

[23] D. Karaboga, "An idea based on honey bee swarm for numerical optimization," Tech. Rep., Technical report-tr06, Erciyes university, engineering faculty, computer engineering department, 2005.

[24] F. Kang, J. Li, and Z. Ma, "Rosenbrock artificial bee colony algorithm for accurate global optimization of numerical functions," Information Sciences, vol. 181, no. 16, pp. 3508-3531, 2011.

[25] A. Banharnsakun, T. Achalakul, and B. Sirinaovakul, "The bestso-far selection in artificial bee colony algorithm," Applied Soft Computing, vol. 11, no. 2, pp. 2888-2901, 2010.

[26] D. Karaboga, B. Gorkemli, C. Ozturk, and N. Karaboga, "A comprehensive survey: artificial bee colony $(\mathrm{ABC})$ algorithm and applications," Artificial Intelligence Review, vol. 42, pp. 21-57, 2014.

[27] J. Ji, W. Pang, Y. Zheng, Z. Wang, and Z. Ma, "A novel artificial bee colony based clustering algorithm for categorical data," PLoS ONE, vol. 10, no. 5, Article ID e0127125, 2015.

[28] O. A. Abdul-Rahman, M. Munetomo, and K. Akama, "An adaptive parameters binary-real coded genetic algorithm for real parameter optimization: Performance analysis and estimation of optimal control parameters," International Journal of Computer Science Issues, vol. 9, no. 2, 2012.

[29] M. Drozdik, H. Aguirre, and K. Tanaka, "Attempt to reduce the computational complexity in multi-objective differential evolution algorithms," in Proceedings of the 2013 15th Genetic and Evolutionary Computation Conference, GECCO 2013, pp. 599606, Amsterdam, The Netherlands, July 2013. 


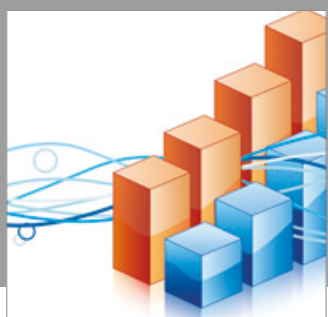

Advances in

Operations Research

vatersals

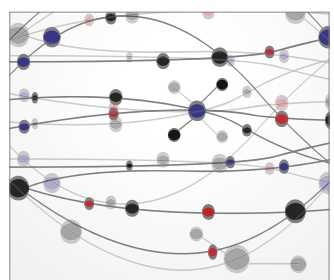

\section{The Scientific} World Journal
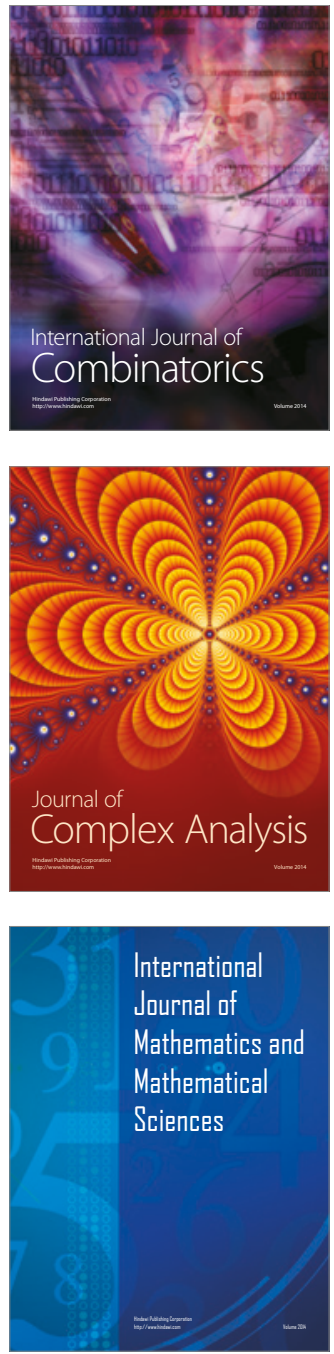
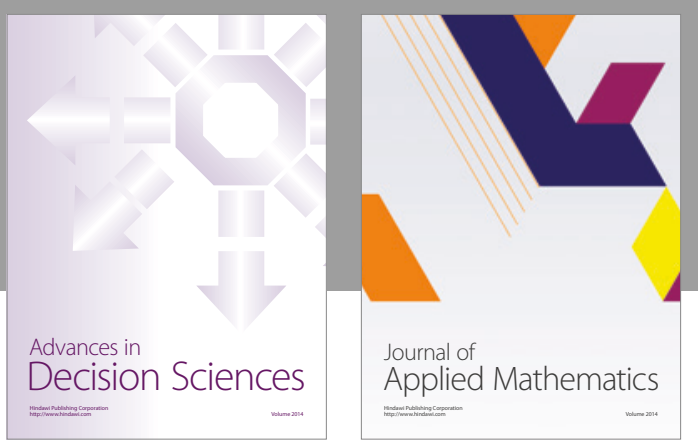

Algebra

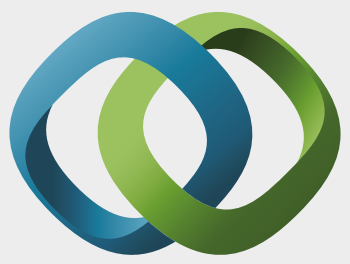

\section{Hindawi}

Submit your manuscripts at

https://www.hindawi.com
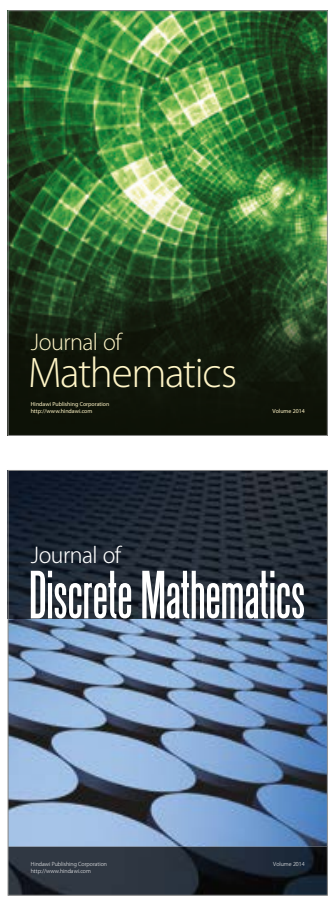

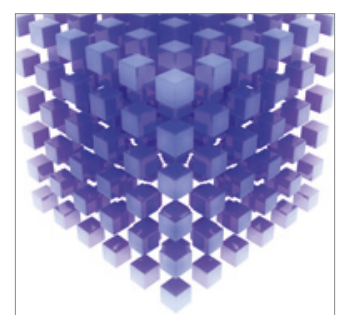

Mathematical Problems in Engineering
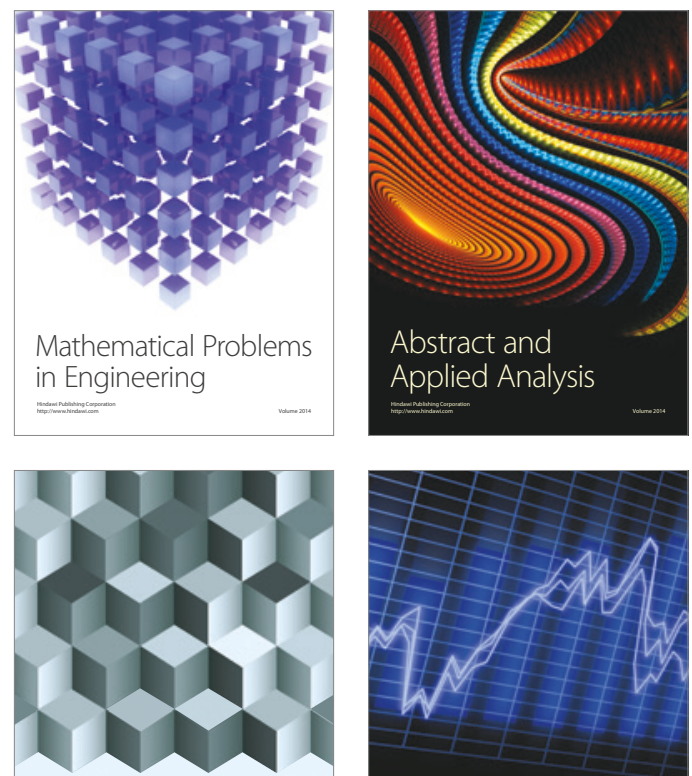

Journal of

Function Spaces

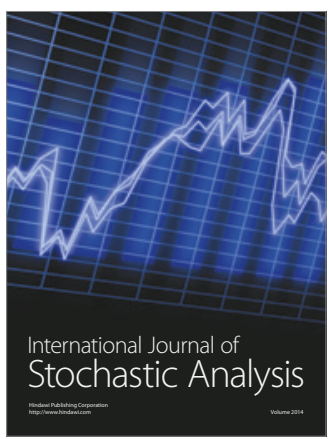

Probability and Statistics
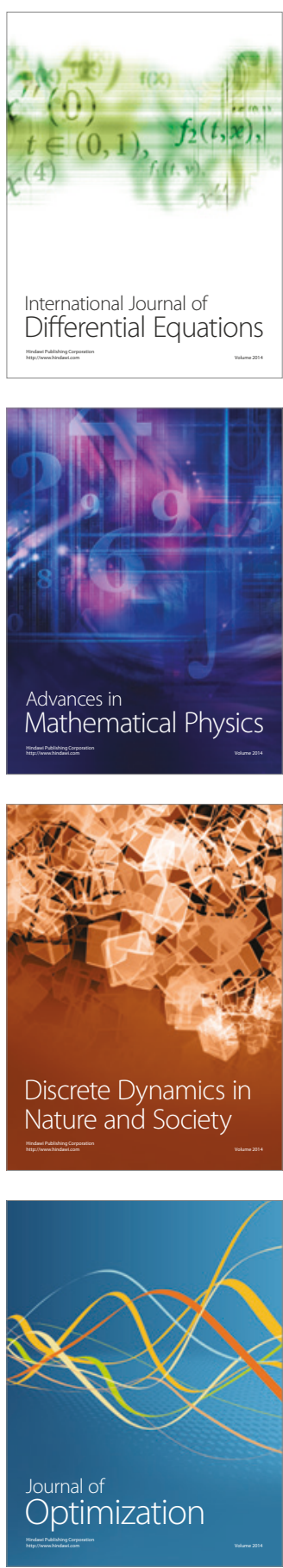